\title{
Analyse par le modèle multiphasique du comportement macroscopique de matériaux renforcés par fibres
}

\author{
Analysis of the macroscopic behaviour of materials reinforced by fibres using a \\ multiphase model
}

\author{
Ghazi Hassen *, Van Tuan Nguyen \\ Laboratoire Navier, École des ponts ParisTech, 6-8, avenue Blaise-Pascal, 77455 Marne-la-Vallée cedex 2, France
}

R É S U M É

Un modèle multiphasique pour l'analyse du comportement macroscopique des matériaux renforcés par fibres est développé dans le cadre d'un comportement élastique linéaire de la matrice et élastique endommageable des renforcements. Ce modèle est ensuite mis en œuvre pour quantifier la réponse macroscopique de structures soumises à des sollicitations simples, pour lesquelles une solution analytique ou semi-analytique peut être développée, mettant en évidence la capacité du modèle à rendre compte du comportement endommageable de tels structures.

\begin{abstract}
A B S T R A C T
A multiphase model is developed for the analysis of the macroscopic behaviour of composite materials constituted of an elastic matrix reinforced with continuously oriented fibres exhibiting a softening behaviour. The model is then performed to develop analytical and semi-analytical solutions for solid structures subjected to uniaxial loadings (oedometric test and traction test) and flexural loading, showing the capacity of the model to quantify the macroscopic softening behaviour of such a composite material.
\end{abstract}

\section{Abridged English version}

Introduction

Fibre-reinforced materials are widely used in the fields of mechanical and civil engineering because of their mechanical performance in terms of stiffness and strength properties. A large variety of these composite artificial materials such as gypsum, fibre-reinforced concrete or fibre-reinforced soils, as well as some natural materials, such as bone tissues, exhibit a microstructure constituted of a matrix and a distribution of continuously oriented fibres.

Unlike artificial composite materials for which the reinforcements exhibit better mechanical properties than the native material, the bone tissues microstructures, is constituted, at a 100-500 nm scale, of an elastic matrix and a distribution of randomly oriented brittle hydroxyapatite (HA) [1]. At the macroscopic scale, this composite material exhibits an elastic

\footnotetext{
* Auteur correspondant.

Adresse e-mail : ghazi.hassen@enpc.fr (G. Hassen).
} 
behaviour followed by a damage phenomenon. The microscopic morphology of gypsum is also constituted of a randomly distributed and elongated brittle crystals that requires the development of a homogenization approach to investigate the macroscopic behavior of such a composite material [2]. Several models are proposed to evaluate a damage variable and to assess the softening behaviour of these composite materials [3-5].

The multiphase approach, developed initially to assess the macroscopic behaviour of soil structures reinforced by linear inclusions in the context of an elastoplastic behaviour of the different constituents is extended here to take into account an infinity of reinforcement phases, each of them representing, at the macroscopic scale, a family of fibres oriented in the same direction. The developed model is then performed to evaluate the macroscopic response of a solid structure subjected to different loadings.

\section{Multiphase description of the fibre-reinforced material}

The multiphase description of a material structure reinforced with a distribution of regularly spaced linear inclusions, oriented with respect to a unit vector $\underline{n}$, consists in replacing the composite material by a superposition of two continuous phases, namely the matrix phase representing the native material and the reinforcement phase representing the distribution of inclusions. These two phases are doted with two different kinematics. In the case of a small volume fraction of reinforcement and a reinforcing material exhibiting very higher mechanical properties as compared to those of the matrix, the macroscopic stress tensor could be expressed as the sum of the partial stresses in the different constituents (1) whereas the macroscopic stiffness tensor is the sum of the matrix stiffness tensor and the uniaxial tensor of the reinforcement phase (2) (see [8] for more details). Under the assumption of perfect bonding between the matrix and the reinforcement phases, the microscopic strains and the macroscopic strain $\underline{E}$ verify the relation (5).

The multiphase description of composite materials composed of an elastic matrix reinforced by elastic brittle fibres continuously oriented in the plane $(O x y)$, consists in replacing the reinforced material by a superposition of a matrix phase and infinity of reinforcement phases representing the different families of parallel fibres. At the macroscopic scale, each phase is perceived as a continuous medium which can afford traction-compression efforts. The macroscopic stress and the macroscopic stiffness tensors are given by (7) and (8) respectively. The combination of the bonding assumption (5) and the constitutive behaviour of the different phases lead to the localisation relationships (9).

In the case of reinforcing fibres exhibiting an elastic behaviour followed by a softening phase [9], the constitutive law of a reinforcement phase could be expressed by (10) where $\alpha_{\mathrm{T}}^{r}$ represents the stiffness of the non-damaged phase whereas $d$ is a damage parameter (Fig. 4).

\section{Illustrative application}

As an application of the multiphase approach developed here, let consider a composite material which microstructure is constituted of a linear elastic matrix and a continuously distributed reinforcing fibres oriented with respect to the plane $0 x y$. This solid structure is subjected to a uniaxial macroscopic deformation (14). The macroscopic response is evaluated analytically in the elastic phase (15) and in the softening phase for elastic-brittle reinforcements $\left(\alpha_{T}^{r}=-\infty\right)(17)-(18)$. The latter phase corresponds to the evolution of a damaged zone, corresponding to the failure of the fibers located in the sector of angles $(-\beta, \beta)$ (Fig. 5). Calculus are performed for different values of the tangent modulus $\alpha_{\mathrm{T}}^{r}$ and the results are drawn in Fig. 6.

The second application of the model corresponds to a traction loading test in plane strain conditions with respect to Oxy. The macroscopic stress and strain tensors are given by (21) and (22). The results of this loading test are drawn on the stress-deformation curve (Fig. 7) for brittle fibres and (Fig. 8) for different values of $\alpha_{T}^{r}$. The curve (Fig. 7) shows three successive phases, the first one corresponds to an elastic evolution which takes end when the horizontal fibres fail. The second phase is a damage phase, in which a sector of angles $(-\beta, \beta)$ is damaged. The third phase takes place when the vertical fibres fail in compression, a second damaged zone appears $(\pi / 2-\gamma, \pi / 2+\gamma)$ and propagates until failure of all the reinforcing fibres.

As a last application, we consider a beam of length $2 \ell$ and height $h=1 \mathrm{~m}$, extending to infinity in the $z$-direction, subjected to a flexural loading applied to its extremities located at $x= \pm \ell$ (Fig. 9(a)). The results are represented in Fig. 9(b) in terms of the evolution of the bending moment per unit transverse length as a function of the curvature. These curves display an elastic behaviour, until the ultimate stress is reached at the superior and inferior extremities of the beam, followed by a softening phase. Since the solution of this boundary value problem corresponds locally to stress and strain fields of the form (21) and (22) (i.e. solution for a plane-strain traction loading), the macroscopic softening of the beam corresponds to the evolution of three damage parameters, the angles $\beta$ and $\gamma$ delimiting the traction and compression damaged zones (Fig. 7) along with a third parameter governing the propagation of the damaged zones from the top and toe of the beam to its axis.

The main interest of the proposed model lies on its capacity to quantify the macroscopic behaviour of composite materials and capture local effects due to the softening-damageable constitutive law of the reinforcing fibres. A F.E.M-based numerical tool incorporating the model could be easily developed where a non local approach should be incorporated to avoid mesh dependency problems. 


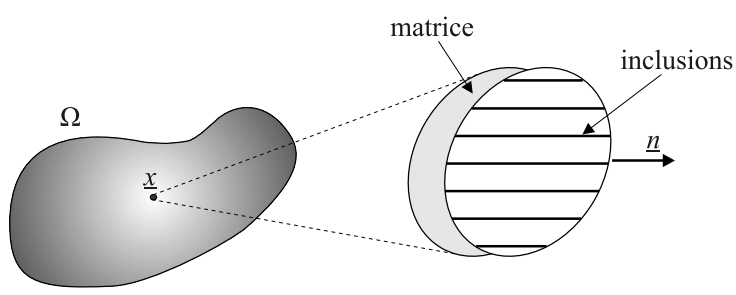

Fig. 1. Description du milieu biphasique.

Fig. 1. Description of the two-phase system.

\section{Introduction}

Les matériaux composites à fibres, apparues dans les années quarante, mettent à profit les excellentes propriétés mécaniques de fibres, et souvent leur faible densité. Ils sont largement utilisés dans les domaines du génie mécanique et du génie civil. Ce type de matériau, tels que béton de fibres, plâtre de fibres ou sols renforcés de fibres, ainsi que certains matériaux naturels, tels que les tissus osseux, présentent une microstructure constituée d'une matrice et d'une distribution de fibres orientées de façon continue dans toutes les directions.

Contrairement aux composites industriels pour lesquels les fibres de renforcement disposent de meilleures propriétés mécaniques que celles du matériau initial, les tissus osseux, disposent à une échelle ne dépassant pas les quelques centaines de nm, d'une microstructure constituée d'une matrice élastique et d'une distribution d'hydroxyapatites (HA) d'orientations aléatoires et dont le comportement est élastique endommageable [1]. Le plâtre pris est un matériau qui s'apparente à cette famille de composites puisqu'il dispose d'une microstructure constituée de cristaux allongés dont le comportement est élastique fragile [2]. Ce type de matériau composite présente un comportement macroscopique élastique suivi d'un phénomène d'endommagement. Plusieurs modèles ont étés proposés afin d'évaluer une variable d'endommagement et de simuler le comportement de ces matériaux composites [3-5].

Une modélisation qualifiée de multiphasique a été récemment proposée pour les ouvrages en sols renforcés par inclusions linéaires dans le contexte d'un comportement élasto-plastique des différents constituants. Ce modèle est ici étendu afin de prendre en compte l'orientation continue des fibres par l'introduction d'une infinité de phases renforcement, chacune d'entre elles représentant, à l'échelle macroscopique, une famille de fibres orientées dans la même direction. Le deuxième volet de ce travail consiste à adopter une loi de comportement de type endommageable avec adoucissement en ce qui concerne les fibres et d'évaluer la réponse macroscopique d'un tel matériau composite soumis à des sollicitations simples telles qu'une déformation macroscopique homogène, un essai de traction et un essai de flexion.

\section{Description du modèle multiphasique du matériau renforcé par fibres}

\subsection{Matériau renforcé selon une seule direction}

Selon le modèle multiphasique [6,7], la description macroscopique d'un matériau renforcé par une famille de fibres orientés selon la même direction $\underline{n}$, consiste à remplacer le matériau composite par la superposition de deux phases continues (milieu biphasique), la phase matrice, représentant le matériau non renforcé, et la phase renforcement représentant la distribution des inclusions (Fig. 1).

La contrainte macroscopique peut alors être exprimée comme la somme des contraintes partielles relatives à chaque phase :

$$
\underline{\underline{\Sigma}}=\underline{\underline{\sigma}}^{m}+\sigma^{r} \underline{n} \otimes \underline{n}
$$

où $\underline{\sigma}^{m}$ désigne la contrainte dans la phase matrice, tandis que $\sigma^{r}$ est la contrainte uniaxiale dans la phase renforcement. Dans le cadre d'un comportement élastique linéaire des différents constituants les lois de comportement de chaque phase s'éxpriment par :

$$
\underline{\underline{\sigma}}^{m}=\mathbb{C}^{m}: \underline{\epsilon}^{m}
$$

en ce qui concerne la phase matrice et :

$$
\sigma^{r}=\alpha^{r} \epsilon^{r}
$$

pour la phase renforcement qui apparaît comme un milieu continu ne pouvant reprendre que des efforts de type tractioncompression. $\mathbb{C}^{m}$ désigne le tenseur des modules élastiques de la phase matrice et $\alpha^{r}$ est la rigidité en traction de la phase renforcement.

Dans le cas où la fraction volumique de renforcement (notée $\eta$ ), définie comme le rapport du volume des renforts sur le volume total du matériau renforcé, est très faible (cas des sols renforcés par inclusion et de certains matériaux composites), 


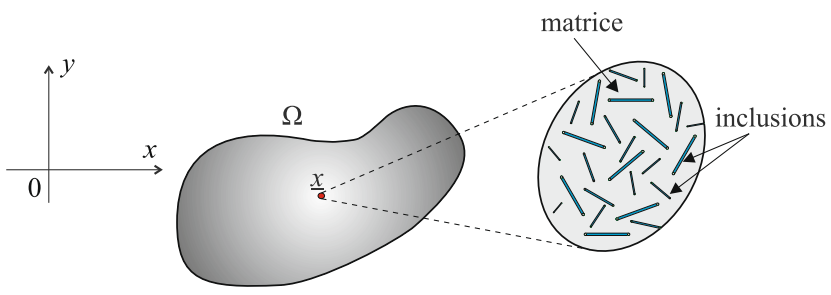

Fig. 2. Microstructure du milieu renforcé.

Fig. 2. Microstructure of the reinforced medium.

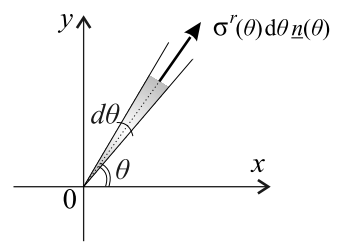

Fig. 3. Efforts internes dans la phase renforcement.

Fig. 3. Internal efforts in the reinforcement phase.

les propriétés mécaniques de la phase matrice $\left(\mathbb{C}^{m}\right)$ sont identifiées à celles de son matériau constitutif tandis que $\alpha^{r}$ est défini comme le produit du module d'Young $E^{r}$ du renfort par la fraction volumique :

$$
\alpha^{r}=\eta E^{r}
$$

Compte tenu de l'hypothèse d'adhérence parfaite les déformations des phases sont reliées à la déformation macroscopique $\underline{\underline{E}}$ par les équations suivantes :

$$
\underline{\underline{E}}=\underline{\underline{\epsilon}}^{m} \text { et } \epsilon^{r}=\underline{\underline{E}}:(\underline{n} \otimes \underline{n})
$$

On en déduit alors le comportement macroscopique du matériau composite exprimé par la relation contrainte-déformation macroscopique $(\underline{\underline{\Sigma}}-\underline{\underline{E}})[8]$

$$
\Sigma=\mathbb{C}^{\text {hom }}: E \quad \text { avec } \mathbb{C}^{\text {hom }}=\mathbb{C}^{m}+\alpha^{r} \underline{n} \otimes \underline{n} \otimes \underline{n} \otimes \underline{n}
$$

$\mathbb{C}^{\text {hom }}$ désigne le tenseur des modules élastiques du milieu biphasique.

\subsection{Matériau renforcé dans toutes les directions du plan Oxy}

On considère maintenant un matériau composite constitué d'une matrice élastique renforcée par des fibres élastiques orientés continûment dans le plan Oxy (Fig. 2).

La modélisation multiphasique de ce matériau composite consiste à décrire le matériau renforcé comme la superposition d'une phase matrice et d'une infinité de phases renforcement, chacune d'entre elles représentant une famille de fibres parallèles. A l'échelle macroscopique, chaque phase est perçue comme un milieu continu. Une démarche analogue à celle adoptée dans [6] pour un nombre fini des phases renforcement permet de relier la contrainte macroscopique aux contraintes par phase comme suit :

$$
\underline{\underline{\Sigma}}=\underline{\sigma}^{m}+\int_{0}^{\pi} \sigma^{r}(\theta) \underline{n}(\theta) \otimes \underline{n}(\theta) \mathrm{d} \theta
$$

où $\sigma^{r}(\theta) \mathrm{d} \theta \underline{n}(\theta)$ désigne le vecteur contrainte élémentaire appliqué sur une facette de normale $\underline{n}(\theta)$ par les fibres de renforcement dont l'orientation est comprise entre les angles $\theta-\frac{\mathrm{d} \theta}{2}$ et $\theta+\frac{\mathrm{d} \theta}{2}$ (Fig. 3). Dans le cadre d'un comportement élastique linéaire de la matrice et des fibres, le comportement macroscopique peut être exprimé par le tenseur $\mathbb{C}^{\text {hom }}$ donné par :

$$
\mathbb{C}^{h o m}=\mathbb{C}^{m}+\int_{0}^{\pi} \alpha^{r}(\theta) \underline{n}(\theta) \otimes \underline{n}(\theta) \otimes \underline{n}(\theta) \otimes \underline{n}(\theta) \mathrm{d} \theta
$$

où $\alpha^{r}(\theta)$ désigne la raideur à la traction-compression des fibres se trouvant dans le secteur compris entre $\left(\theta-\frac{\mathrm{d} \theta}{2}\right)$ et $\left(\theta+\frac{\mathrm{d} \theta}{2}\right)$ (Fig. 3). 


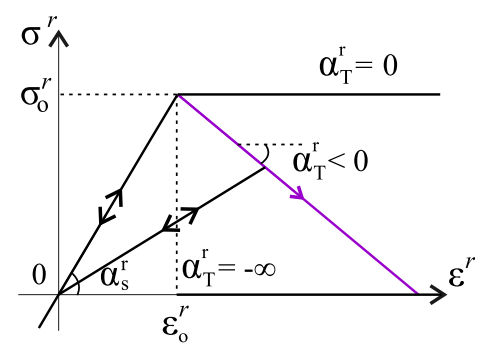

Fig. 4. Courbe contrainte-déformation des phases renforcements.

Fig. 4. Stress-strain curve of the reinforcement phases.

Sous l'hypothèse d'une adhérence parfaite entre la phase matrice et les phases renforcement (i.e. $\underline{\underline{E}}=\underline{\underline{\epsilon}}^{m}$ et $\epsilon^{r}(\theta)=\underline{\underline{E}}$ : $(\underline{n}(\theta) \otimes \underline{n}(\theta)))$ les contraintes dans les différentes phases peuvent être évaluées par les équations de localisation suivantes :

$$
\left\{\begin{array}{l}
\underline{\underline{\sigma}}^{m}=\mathbb{C}^{m}:\left(\mathbb{C}^{\text {hom }}\right)^{-1}: \underline{\underline{\Sigma}} \\
\sigma^{r}(\theta)=\alpha^{r}(\theta)\left(\left(\mathbb{C}^{h o m}\right)^{-1}: \underline{\underline{\Sigma}}\right):(\underline{\underline{n}}(\theta) \otimes \underline{n}(\theta)) \mathrm{d} \theta
\end{array}\right.
$$

\subsection{Comportement élastique endommageable des fibres}

L'état des phases renforcement est caractérisé par deux variables, la déformation $\epsilon^{r}$ et l'endommagement quantifié par une variable scalaire $d$ comprise entre 0 et 1 [9]. La relation contrainte-déformation dans chaque phase renforcement s'écrit :

$$
\sigma^{r}=\alpha^{r}(d) \epsilon^{r} \quad \text { où } \alpha^{r}(d)=(1-d) \alpha_{s}^{r} ; d \text { croissant }
$$

où $\alpha_{s}^{r}$ est la rigidité à l'état sain. Il est aussi possible de décrire le comportement adoucissant des phases renforcement, en renseignant le module tangent $\alpha_{\mathrm{T}}^{r}$ et la contrainte au pic $\sigma_{o}^{r}$ (Fig. 4). Dans le cas où la valeur $\alpha_{\mathrm{T}}^{r}$ vaut 0 le comportement élastique du matériau sain est suivi d'un plateau horizontal, tandis que lorsque $\alpha_{\mathrm{T}}^{r}$ tend vers $-\infty$, le comportement devient élastique fragile. Dans ce cas, la loi de comportement de la phase renforcement représentant les fibres d'orientation $\theta$ s'écrit :

$$
\sigma^{r}(\theta)= \begin{cases}\alpha^{r}(\theta) \epsilon^{r}(\theta) & \text { si }\left|\epsilon^{r}(\theta)\right| \leqslant \epsilon_{o}^{r}(d)=\frac{\sigma_{o}^{r}(d)}{\alpha_{s}^{r}(d)} \\ 0 & \text { sinon }\end{cases}
$$

avec

$$
\sigma_{o}^{r}(d)-\sigma_{o}^{r}=\alpha_{\mathrm{T}}^{r}\left[\epsilon_{o}^{r}(d)-\epsilon_{o}^{r}\right]
$$

où $\epsilon_{o}^{r}$ désigne la déformation correspondante à la limite d'élasticité initiale $\sigma_{o}^{r}$ tandis que $\epsilon_{o}^{r}(d)$ est la déformation correspondante à la limite d'élasticité $\sigma_{0}^{r}(d)$ de la phase renforcement pour la valeur du paramètre d'endommagement $d$.

\section{Application illustrative}

\subsection{Cas d'une déformation macroscopique uniaxiale (essai œedométrique)}

Comme une première application du modèle, on considère un matériau composite dont la microstructure est constituée d'une matrice élastique linéaire et d'une distribution isotrope continue dans le plan (Oxy) de fibres de renforcement élastiques fragiles. En calculant les termes de $\mathbb{C}^{\text {hom }}$ donné par (6), la loi de comportement macroscopique du matériau composite peut être exprimée, en utilisant la notation de Voigt par :

$$
\left[\begin{array}{c}
\Sigma_{\mathrm{xx}} \\
\Sigma_{\mathrm{yy}} \\
\Sigma_{\mathrm{zz}} \\
\Sigma_{\mathrm{yz}} \\
\Sigma_{\mathrm{xz}} \\
\Sigma_{\mathrm{xy}}
\end{array}\right]=\left[\begin{array}{cccccc}
\lambda+2 \mu+\frac{3 \pi}{8} \alpha_{s}^{r} & \lambda+\frac{\pi}{8} \alpha_{s}^{r} & \lambda & 0 & 0 & 0 \\
\lambda+\frac{\pi}{8} \alpha_{s}^{r} & \lambda+2 \mu+\frac{3 \pi}{8} \alpha_{s}^{r} & \lambda & 0 & 0 & 0 \\
\lambda & \lambda & \lambda+2 \mu & 0 & 0 & 0 \\
0 & 0 & 0 & \mu & 0 & 0 \\
0 & 0 & 0 & 0 & \mu & 0 \\
0 & 0 & 0 & 0 & 0 & \mu+\frac{\pi}{8} \alpha_{s}^{r}
\end{array}\right]\left[\begin{array}{c}
E_{\mathrm{xx}} \\
E_{\mathrm{yy}} \\
E_{\mathrm{zz}} \\
2 E_{\mathrm{yz}} \\
2 E_{\mathrm{xz}} \\
2 E_{\mathrm{xy}}
\end{array}\right]
$$




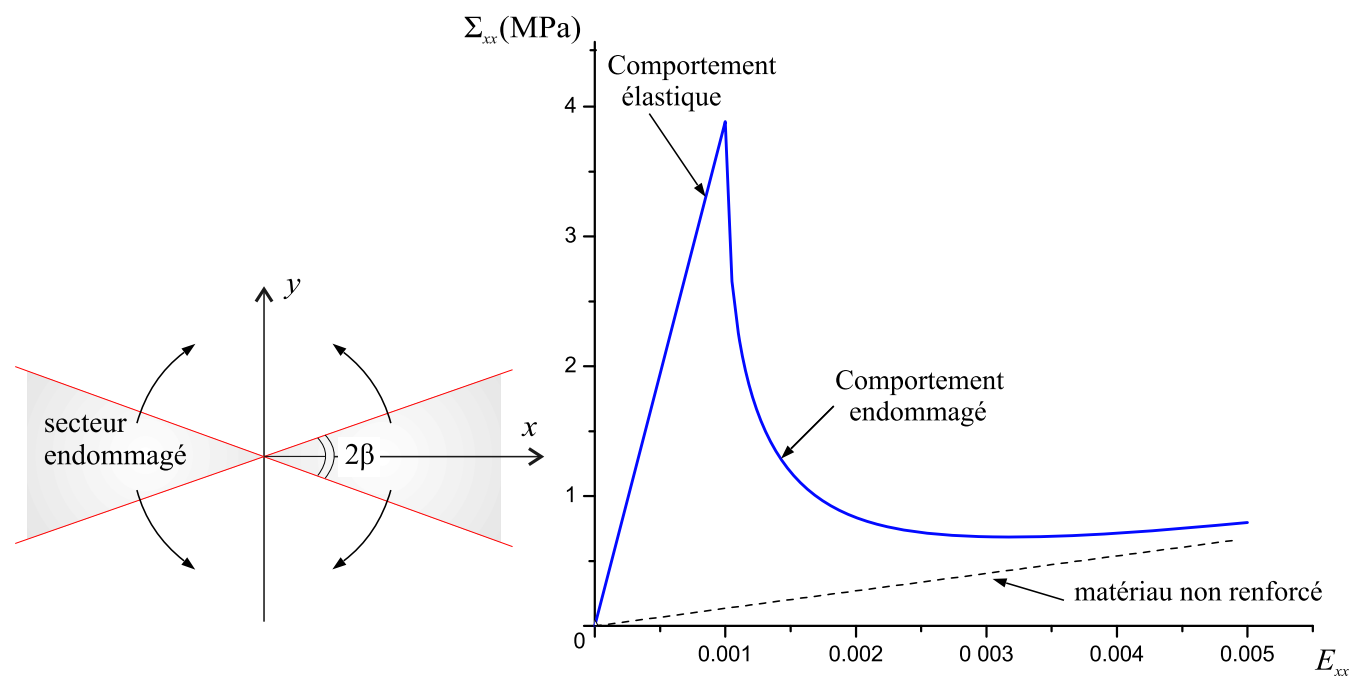

(a)

(b)

Fig. 5. (a) Evolution de la zone endommagée. (b) Comportement macroscopique (déformation uniaxiale).

Fig. 5. (a) Evolution of the damaged zone. (b) Macroscopic behaviour (uniaxial macroscopic strain).

Considérons maintenant le cas où un tel matériau composite est soumis à une déformation homogène et uniaxiale de la forme :

$$
\underline{\underline{E}}=E_{x x} \underline{e}_{x} \otimes \underline{e}_{x}
$$

Dans un premier temps, tous les constituants du matériau composite (matrice et renforcements) sont élastiques, la contrainte macroscopique suivant l'axe $(O x)$ est déterminée par la loi de comportement macroscopique (13); elle s'écrit :

$$
\Sigma_{x x}=\left(\lambda+2 \mu+\frac{3 \pi}{8} \alpha_{s}^{r}\right) E_{x x}
$$

Cette relation reste valable jusqu'à ce que le critère soit atteint dans les fibres orientées suivant la direction $(O x)$ ce qui correspond à la limite d'élasticité :

$$
E_{x x}^{e}=\epsilon_{0}^{r}
$$

Supposons que le comportement des fibres est fragile (module tangent $\alpha_{\mathrm{T}}^{r}=-\infty$ ) et poursuivons le chargement au delà de cette limite d'élasticité $\left(E_{x x}>E_{x x}^{e}\right)$. Un secteur angulaire de fibres endommagées, symétrique par rapport à l'axe de sollicitation $O x$, comprises entre les directions d'angle $-\beta$ et $\beta$ apparaît (Fig. 5(a)). La contrainte macroscopique s'exprime en fonction de ce paramètre d'endommagement $\beta$ par :

$$
\Sigma_{x x}=[\lambda+2 \mu+f(\beta)] E_{x x}
$$

avec :

$$
f(\beta)=\alpha_{s}^{r}\left(\frac{3 \pi}{8}-\frac{\cos ^{3} \beta \sin \beta}{2}-\frac{3 \cos \beta \sin \beta}{4}-\frac{3 \beta}{4}\right)
$$

où

$$
\beta=\operatorname{arcos}\left(\sqrt{\frac{\sigma_{0}^{r}}{\alpha_{s}^{r} E_{x x}}}\right)
$$

$f(\beta)$ représente le gain en raideur apporté par les renforcements qui tend vers 0 lorsque $\beta$ tend vers $\frac{\pi}{2}$ (i.e. $E_{x x} \rightarrow \infty$ ).

Le résultat est présenté sur la Fig. 5(b), sous la forme d'une courbe donnant pour l'évolution de la contrainte macroscopique $\Sigma_{x x}$ en fonction de la déformation imposée $E_{x x}$, obtenue pour les caractéristiques suivantes :

$$
E^{m}=100 \mathrm{MPa}, \quad v^{m}=0.3
$$

pour la phase matrice et

$$
\alpha_{s}^{r}=100 \mathrm{MPa}, \quad \sigma_{o}^{r}=0.1 \mathrm{MPa}
$$

pour les phases renforcement. 


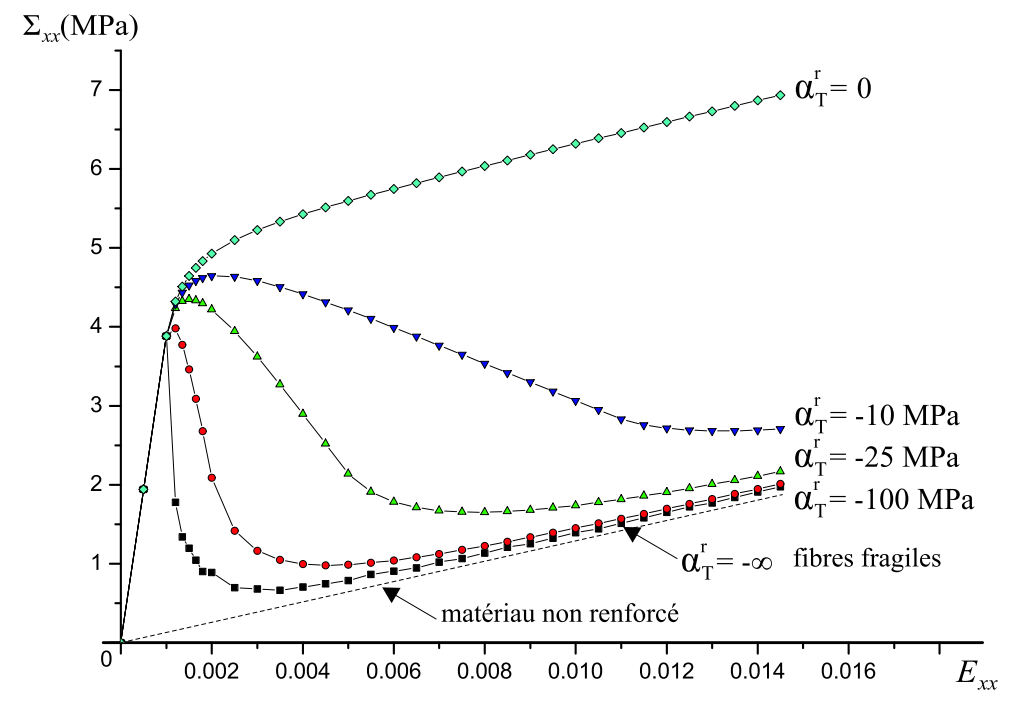

Fig. 6. Déformation macroscopique uniaxiale : Courbes contrainte-déformation en fonction de $\alpha_{\mathrm{T}}^{r}$.

Fig. 6. Macroscopic uniaxial strain: Stress-strain curves for different values of $\alpha_{\mathrm{T}}^{r}$.

Cette courbe présente une première phase élastique linéaire (15) qui se termine lorsque la limite d'élasticité (16) est atteinte. Au-delà de cette valeur, une zone endommagée se propage et la courbe tend asymptotiquement vers le comportement de la matrice seule.

Ce problème est repris pour des différentes valeurs de $\alpha_{\mathrm{T}}^{r}$. Les résultats sont représentés sur la Fig. 6 . Mis à part les deux courbes extrêmes correspondantes aux fibres fragiles $\left(\alpha_{\mathrm{T}}^{r}=-\infty\right)$ et ductiles $\left(\alpha_{\mathrm{T}}^{r}=0\right)$ le processus d'endommagement de la structure sous chargement de déformation macroscopique homogène uniaxiale est à deux niveaux : endommagement local des phases renforcement caractérisé par la variable scalaire $d$ introduite en (10) et un endommagement global exprimé par l'évolution de l'angle $\beta$ caractérisant la zone des fibres où le critère est saturé. Ces courbes tendent asymtotiquement vers la courbe correspondant au comportement de la matrice.

\subsection{Cas d'une sollicitation de traction en déformation plane}

On considère maintenant un éprouvette constituée du même matériau composite qu'en Section $3.1\left(\alpha_{\mathrm{T}}^{r}=-\infty\right)$. Cette éprouvette est soumise à un essai de traction, suivant la direction $x$, en déformation plane dans le plan Oxy. L'état de contrainte macroscopique est de la forme :

$$
\underline{\underline{\Sigma}}=\Sigma_{x x} \underline{e}_{x} \otimes \underline{e}_{x}+\Sigma_{z z} \underline{e}_{z} \otimes \underline{e}_{z}
$$

Tandis que la déformation macroscopique suivant $z$ est nulle $\left(E_{z z}=0\right)$ :

$$
\underline{\underline{E}}=E_{x x} \underline{e}_{x} \otimes \underline{e}_{x}+E_{y y} \underline{e}_{y} \otimes \underline{e}_{y}
$$

Considérons les mêmes caractéristiques mécaniques que l'exemple précédent (19) et (20), les résultats de cette simulation sont représentés sur la Fig. 7 donnant l'évolution de $\Sigma_{x x}$ en fonction de $E_{x x}$.

Cette courbe montre trois phases successives, la première phase correspond à une évolution élastique qui prend fin lorsque la contrainte dans la phase renforcement, représentant la famille de fibres horizontales, atteint la valeur limite $\sigma_{0}^{r}$. La deuxième phase est une phase d'endommagement, qui correspond à l'évolution du secteur de demi-angle $\beta$ d'une manière analogue à celle décrite en Section 3. La troisième phase apparaît lorsque les fibres verticales cassent en compression (secteur de demi-angle $\gamma$ ) par effet Poisson. Une deuxième zone endommagée apparaît alors et se propage jusqu'à l'endommagement de toutes les fibres de renforcement. Il est à préciser que le modèle peut être appliqué pour le cas de fibres souples (raideur en compression nulle), la troisième phase n'aura dans ce cas plus lieu.

Dans le cas où les fibres de renforcement sont élastiques-endommageables, la réponse de l'éprouvette est représenté sur la Fig. 8 pour différentes valeurs de $\alpha_{\mathrm{T}}^{r}$.

\subsection{Cas d'une poutre soumise à un chargement de flexion en déformation plane}

La $3^{\text {ème }}$ application du modèle a pour objectif de développer la relation moment-courbure d'une poutre en matériau composite endommageable soumise à un essai de flexion en déformation plane. 


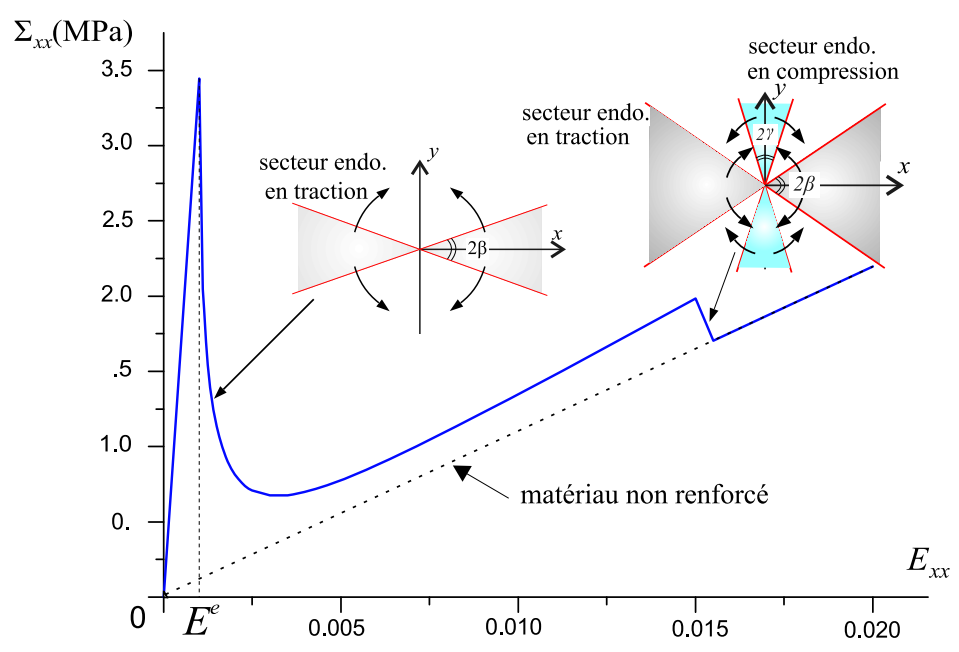

Fig. 7. Courbe contrainte-déformation : essai de traction en déformation plane.

Fig. 7. Stress-strain curve: traction test in plane strain conditions.

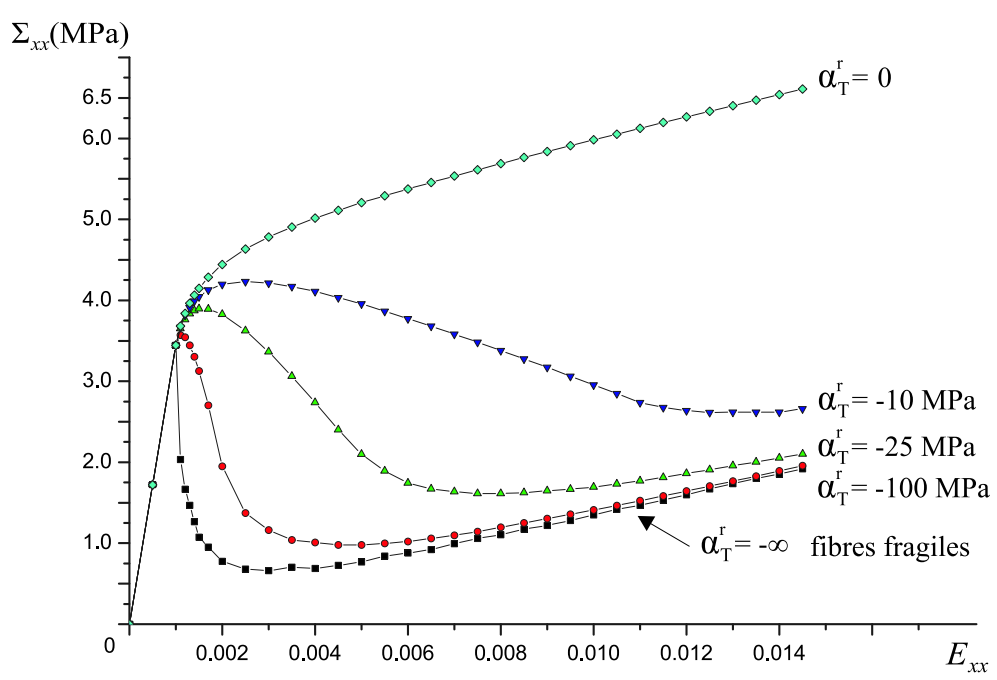

Fig. 8. Essai de traction en déformation plane : Courbes contrainte-déformation en fonction de $\alpha_{\mathrm{T}}^{r}$.

Fig. 8. Traction test in plane strain conditions: Stress-strain curves for different values of $\alpha_{\mathrm{T}}^{r}$.

On considère une poutre de longueur $2 \ell$ et de hauteur $2 h$, sollicitée en déformation plane dans le plan Oxy par l'application d'une rotation $\omega$ à ces deux extrémités $x= \pm \ell$ (Fig. 9(a)). Le champ de déformation solution de ce problème est invariant le long de l'axe de la poutre, il correspond localement à une sollicitation de traction-compression en déformation plane (22) pour lequel le paramètre de chargement est $\frac{\omega}{\ell}$.

La poutre présente une réponse élastique linéaire jusqu'à la valeur $\chi^{e}$ de la courbure :

$$
\chi^{e}=\frac{\omega^{e}}{\ell}=\frac{\epsilon_{0}^{r}}{h}
$$

qui correspond à l'endommagement des fibres horizontales des deux âmes, supérieure (en compression) et inférieure (en traction), de la poutre.

Au delà de cette limite d'élasticité un mécanisme d'endommagement à plusieurs niveaux a lieu qui correspondent à l'évolution de la zone endommagée depuis les deux bords supérieur et inférieur de la poutre vers son axe, ainsi qu'à une évolution locale des angles $\beta$ et $\gamma$ (voir Fig. 7).

Les résultats obtenus en considérant les caractéristiques (19) et (20) sont représentés sur la Fig. 9(b) pour différentes valeurs de $\alpha_{\mathrm{T}}^{r}$. 


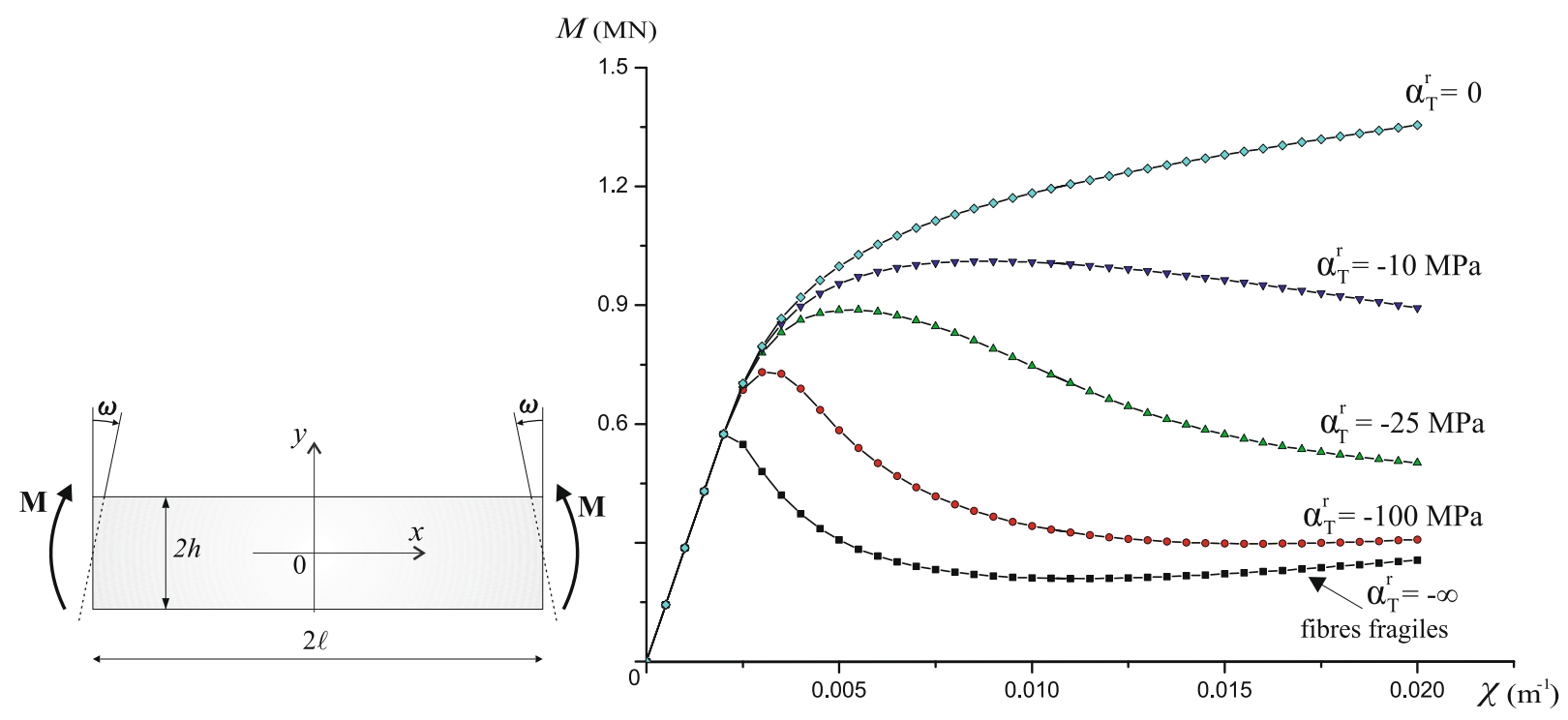

(a)

(b)

Fig. 9. (a) Géométrie de la poutre. (b) Courbes moment-courbure.

Fig. 9. (a) Flexural loading. (b) Bending-curvature curves.

\section{Conclusion}

Un modèle multiphasique est proposé qui permet de décrire le comportement macroscopique des matériaux renforcés par fibres. Ce modèle a été étendu pour prendre en compte un comportement élastique endommageable des fibres et leur orientation continue dans l'espace. Un des avantages majeurs d'une telle approche réside dans la simplicité de la mise en œuvre de la méthode permettant d'avoir des solutions analytiques ou semi-analytique tels que celles développées en Section 3. Le développement d'un outil de calcul numérique utilisant la méthode des éléments finis par exemple, serait très utile pour l'analyse du comportement macroscopique de structures en matériau composite à fibres soumises à un chargement cyclique et multiaxial. Une approche non locale permettrait de passer outre les problèmes numériques de localisation dûs à l'adoucissant des phases renforcements.

Le positionnement de ce travail par rapport aux études antérieures [1,2], dans lesquels des approches de type "Eshelby " sont utilisées constitue la suite de ce travail dont les premiers résultats, obtenus dans le cadre d'un comportement élastique des différents constituants, montrent que les différentes méthodes convergent vers la même estimation du comportement macroscopique du matériau composite.

\section{Références}

[1] A. Fritsch, Interprétentation multi-échelle de l'élasticité et de la résistance de l'os et des biomatériaux en hydroxyapatite, vitrocéramique ou titane : une approche basée sur la micromécanique des milieux continus, Thèse doctorat, ENPC, 2009.

[2] J. Sanahuja, L. Dormieux, S. Meille, C. Hellmich, A. Fritsch, Micromechanical explanation of elasticity and strength of gypsum: From elongated anisotropic crystals to isotropic porous polycrystals, J. Eng. Mech. 136 (2010) 239.

[3] J. Lemaire, J.L. Chaboche, A new formulation of continuum damage mechanics (CDM) for composite materials, Aerospace Sci. Technol. 1 (4) (1997) $247-257$.

[4] J.P. Cordebois, F. Sidoroff, Anisotropie élastique induite par endommagement, in : Colloque Euromech 115, Editions du CNRS, no. 395, Grenoble, 1979.

[5] J.L. Chaboche, Le concept de contrainte effective appliquée à l'élasticité et à la viscoplasticité en présence d'un endommagement anisotrope, in : Colloque Euromech 115, Editions du CNRS, Grenoble, 1979, pp. 737-760.

[6] P. de Buhan, B. Sudret, Modélisation multiphasique de matériaux renforcés par inclusions linéaires, C. R. Acad. Sci. Paris, Ser. IIb 327 (1999) 7-12.

[7] B. Sudret, Modélisation multiphasique des ouvrages renforcés par inclusions, Thèse doctorat, ENPC, 1999.

[8] P. de Buhan, G. Hassen, Multiphase approach as a generalized homogenization procedure for modelling the macroscopic behaviour of soils reinforced by linear inclusions, Eur. J. Mech. A Solids 27 (2008) 662-679.

[9] E. Lorentz, Lois de comportement a gradients de variables internes construction, formulation variationnelle et mise en œuvre numérique, Thèse doctorat, Université Paris 6, 1999. 